\title{
Accuracy of diagnostic testing in primary ciliary dyskinesia
}

\author{
Claire L. Jackson 1,2,7, Laura Behan 1,2,3,4,7, Samuel A. Collins ${ }^{1,2,3}$, \\ Patricia M. Goggin ${ }^{1,5}$, Elizabeth C. Adam ${ }^{1,2}$, Janice L. Coles ${ }^{1,2}$, Hazel J. Evans ${ }^{1,3}$, \\ Amanda Harris ${ }^{1,3}$, Peter Lackie ${ }^{1,2,5}$, Samantha Packham ${ }^{1,3}$, Anton Page ${ }^{1,5}$, \\ James Thompson ${ }^{1,2}$, Woolf T. Walker ${ }^{1,2,3}$, Claudia Kuehni ${ }^{6}$ and \\ Jane S. Lucas ${ }^{1,2,3}$
}

Affiliations: ${ }^{1}$ Primary Ciliary Dyskinesia Centre, University Hospital Southampton NHS Foundation Trust, Southampton, UK. ${ }^{2}$ Clinical and Experimental Sciences Academic Unit, University of Southampton Faculty of Medicine, Southampton, UK. ${ }^{3}$ NIHR Southampton Respiratory Biomedical Research Unit, University of Southampton and University Hospital Southampton NHS Foundation Trust, Southampton, UK. ${ }^{4}$ Department of Applied Psychology, University College Cork, Cork, Ireland. ${ }^{5}$ Biomedical Imaging Unit, University of Southampton Faculty of Medicine and University Hospital Southampton NHS Foundation Trust, Southampton, UK. ${ }^{6}$ Institute of Social and Preventive Medicine, University of Bern, Bern, Switzerland. ${ }^{7}$ Both authors contributed equally.

Correspondence: Jane S. Lucas, Primary Ciliary Dyskinesia Centre, University Hospital Southampton NHS Foundation Trust, Tremona Road, Southampton S016 6YD, UK. E-mail: jlucas1dasoton.ac.uk

ABSTRACT Diagnosis of primary ciliary dyskinesia (PCD) lacks a "gold standard" test and is therefore based on combinations of tests including nasal nitric oxide (nNO), high-speed video microscopy analysis (HSVMA), genotyping and transmission electron microscopy (TEM). There are few published data on the accuracy of this approach.

Using prospectively collected data from 654 consecutive patients referred for PCD diagnostics we calculated sensitivity and specificity for individual and combination testing strategies. Not all patients underwent all tests.

HSVMA had excellent sensitivity and specificity (100\% and 93\%, respectively). TEM was 100\% specific, but $21 \%$ of PCD patients had normal ultrastructure. $\mathrm{nNO}\left(30 \mathrm{~nL} \cdot \mathrm{min}^{-1}\right.$ cut-off) had good sensitivity and specificity (91\% and 96\%, respectively). Simultaneous testing using HSVMA and TEM was 100\% sensitive and $92 \%$ specific.

In conclusion, combination testing was found to be a highly accurate approach for diagnosing PCD. HSVMA alone has excellent accuracy, but requires significant expertise, and repeated sampling or cell culture is often needed. TEM alone is specific but misses $21 \%$ of cases. $\mathrm{nNO}\left(\leqslant 30 \mathrm{~nL} \cdot \mathrm{min}^{-1}\right)$ contributes well to the diagnostic process. In isolation nNO screening at this cut-off would miss $\sim 10 \%$ of cases, but in combination with HSVMA could reduce unnecessary further testing. Standardisation of testing between centres is a future priority.

@ERSpublications

Combination testing in PCD diagnosis remains the most accurate approach, but standardisation is needed http://ow.ly/TLEDu

Editorial comment in: Eur Respir J 2016; 47: 699-701 [DOI: 10.1183/13993003.01914-2015].

This article has supplementary material available from erj.ersjournals.com

Received: May 132015 | Accepted after revision: Oct 152015 | First published online: Dec 032015

Support statement: The National Primary Ciliary Dyskinesia (PCD) Diagnostic Service at University Hospital Southampton is commissioned and funded by NHS England. Research at Southampton PCD Centre receives funding from EU-FP7 BESTCILIA 305404. PCD research in Southampton is supported by the National Institute for Health Research (NIHR) Southampton Respiratory Biomedical Research Unit and the NIHR Wellcome Trust Clinical Research Facility. J.S. Lucas, C. Kuehni, W.T. Walker and L. Behan are members of the European Respiratory Society Task Force to develop a practice guideline for diagnosis of PCD (ERS TF-2014-04). Authors are participants in COST Action BEAT-PCD (BM1407). Funding information for this article has been deposited with FundRef.

Conflict of interest: Disclosures can be found alongside the online version of this article at erj.ersjournals.com

Copyright $\odot$ ERS 2016. ERJ Open articles are open access and distributed under the terms of the Creative Commons Attribution Non-Commercial Licence 4.0. 


\section{Introduction}

Recent advances in the diagnosis of patients with primary ciliary dyskinesia (PCD) have included networks of specialists developing protocol-driven testing [1-4], international consensus guidelines [5] and rapid expansion of known PCD-related genes [3].

There is no "gold-standard" test for PCD, hence European consensus guidelines (2009) [5] recommend a combination of tests including nasal nitric oxide (nNO) screening [4, 6], high-speed video microscopy analysis (HSVMA) of ciliary beat frequency (CBF) and pattern (CBP) [7-10] and transmission electron microscopy (TEM) analysis of ciliary ultrastructure [11, 12]. Reanalysis following submerged [13] or airliquid interface (ALI) [14] culture may be useful to exclude secondary ciliary dyskinesia or confirm PCD when analysis of the primary sample is abnormal, and may provide additional cilia if the primary sample is inadequate. The 2009 guidelines [5] also suggest potential adjuncts to diagnosis including immunofluorescence labelling of cilia proteins [15], pulmonary radioaerosol mucociliary clearance [16, 17] and genotyping. Since 2009 there have been rapid advances in the discovery of genes responsible for PCD $[1,3]$, thus allowing genetic testing to take a prominent position in some countries, but it is currently not funded in the English public healthcare system. The English PCD service $[1,18]$ diagnoses PCD using nNO, HSVMA and TEM, with reanalysis following ALI culture for inconclusive and positive samples.

Several articles have reported the accuracy of individual tests for the diagnosis of PCD, but none have considered all available diagnostic data. Most reports have failed to include the significant numbers of "inconclusive" results [19]. The aim of this study was to determine the accuracy of PCD diagnostic tests (nNO, HSVMA and TEM) when used singularly or in combination, based on a large prospective study of consecutive patients referred for diagnostic testing.

\section{Methods}

Local and national research and development and ethical approvals were obtained (Southampton and South West Hampshire research ethics 07/Q1702/109).

\section{Participants}

868 consecutive subjects were referred to the national PCD centre at University Hospital Southampton (UHS) for diagnostic testing between 2007 and 2013; 654 had adequate data and samples for inclusion. The population served by the centre is predominantly Caucasian and nonconsanguineous. Patients attended UHS or samples were couriered to UHS from satellite referral centres, with no prescreening of $\mathrm{nNO}$.

\section{Diagnostic testing}

The pathway leading to diagnostic outcomes is summarised in figure 1. Details of the method are provided in the online supplementary material.

\section{Patients and samples}

Patients were required to have been free of infection for $\geqslant 4$ weeks. At UHS, demographic and clinical history was recorded using a standard form. At UHS nNO was measured [20] using a chemiluminescence analyser (NIOX Flex; Aerocrine, Solna, Sweden) aspirating nasal air from the nostril at $0.3 \mathrm{~L} \cdot \mathrm{min}^{-1}$ during a breath-hold manoeuvre. Based on experience, since 2007 we have considered an arbitrary cut-off of $\leqslant 30 \mathrm{~nL} \cdot \mathrm{min}^{-1}$. Following nNO measurement, a nasal brush biopsy provided epithelial cells for HSVMA, TEM and ALI culture.

Satellite centres completed patient proformas and brush biopsies were couriered to UHS. Cells for HSVMA and ALI culture were transported in buffered medium within $3 \mathrm{~h}$, while fixed samples for TEM were accepted with longer transportation times.

\section{Laboratory analyses}

HSVMA and TEM were analysed in blinded fashion by PCD-specialist microscopists (online supplementary material). At least six healthy strips of ciliated epithelium were recorded at 500 frames per second (fps). Sequences were played back at $30 \mathrm{fps}$ to observe the CBP and calculate CBF. CBP was qualitatively assessed as normal, dyskinetic (static, uncoordinated, rotational, reduced beat amplitude, slow or hyperfrequent), valid-inconclusive despite adequate sample or invalid-inconclusive due to inadequate sample. HSVMA was only reported normal if both CBF (normal range 11-20 Hz) and CBP were normal.

TEM analysis was carried out if HSVMA was abnormal or inconclusive. $\geqslant 100$ cilia were imaged in transverse section at $\times 60000$ magnification for the assessment of axonemal structure. Using in-house normative data, quantitative analysis determined ciliary ultrastructure as normal, abnormal, valid-inconclusive or 


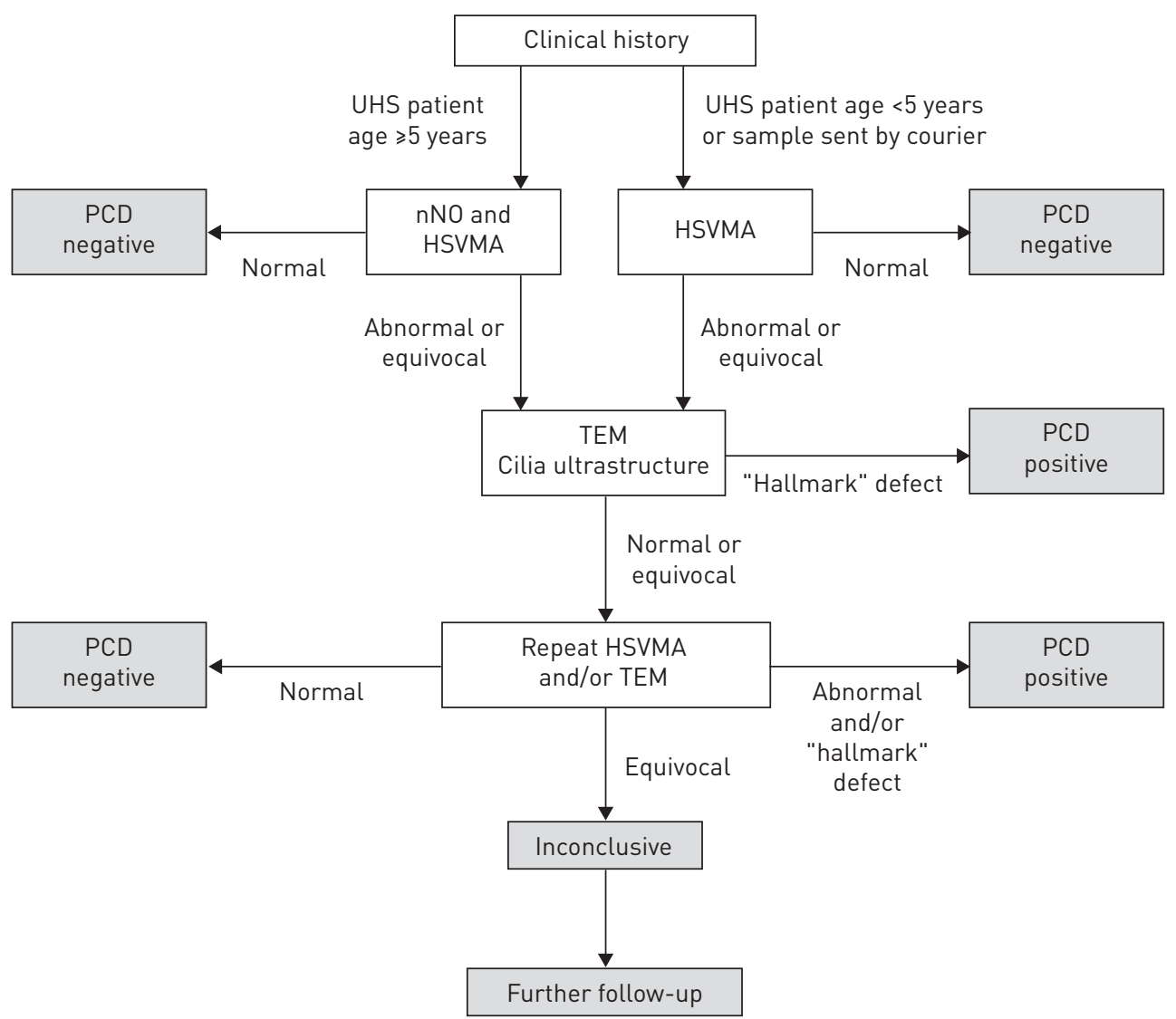

FIGURE 1 Primary ciliary dyskinesia (PCD) diagnostic pathway for patients and samples. Diagnostic tests included nasal nitric oxide (nNO), high-speed video microscopy analysis (HSVMA) and transmission electron microscopy (TEM). Not all patients underwent all tests. UHS: University Hospital Southampton.

inadequate-inconclusive. HSVMA and/or TEM were reanalysed following ALI culture or repeat biopsy unless results were normal.

\section{Diagnostic decisions}

Data were reviewed at multidisciplinary team meetings, attended by a clinician, an HSVMA microscopist and a TEM microscopist. All clinical and diagnostic data were considered when agreeing the diagnostic outcome as PCD-positive, PCD-negative or inconclusive.

Positive diagnosis was reported in patients with typical clinical history, usually with at least two abnormal diagnostic tests (TEM, HSVMA and nNO), but in patients with a strong history (e.g. sibling with PCD or "full" clinical phenotype (e.g. neonatal respiratory distress at term followed by daily wet cough, persistent rhinitis and glue ear, often associated with episodes of upper and lower respiratory tract infection)), we occasionally reported a positive diagnosis based on "hallmark" TEM or repeated HSVMA consistent with PCD. CBP was considered positive if the pattern was typical of PCD rather than secondary ciliary dyskinesia, determined either from two brushing biopsies or from one brushing biopsy with reanalysis following ALI culture.

Negative diagnosis was reported if 1) HSVMA with or without TEM was normal or 2) HSVMA and TEM abnormalities were consistent with secondary rather than primary dyskinesia and normal nNO (if available).

A valid-inconclusive diagnosis was reported if, on repeated testing, adequate samples had subtle abnormalities not "classical" for PCD but outside the range of our experience of secondary defects. It was considered that these patients might have subtle or rare variants of ciliary phenotype. Patients were therefore told that the diagnosis was equivocal, with the recommendation that they received appropriate treatment (e.g. airway clearance or treatment of exacerbations). They were investigated for other causes of their symptoms (e.g. cystic fibrosis genotype and immunology) and were kept under review for further testing as new tests become available (e.g. new PCD-associated mutations). 
If TEM and HSVMA were inconclusive due to inadequate samples, e.g. sparse cilia, the diagnostic outcome was invalid-inconclusive and patients were invited for repeat testing. Patients with normal TEM in isolation were considered invalid-inconclusive, since TEM misses $20-30 \%$ of PCD cases [21]. Patients with nNO $\leqslant 30 \mathrm{~nL} \cdot \mathrm{min}^{-1}$ were deemed likely to have PCD, but it was never accepted as a lone diagnostic test.

\section{Statistical analysis}

Data were prospectively recorded in an Access database (Microsoft, Redmond, WA, USA) and exported to SPSS Statistics 21 (IBM Corp., Armonk, NY, USA) and GraphPad Prism 6 (La Jolla, CA, USA) for analysis. Additional details are listed in the online supplementary material.

The distribution of clinical data were examined by univariate analysis. Prevalence of categorical variables was presented as percentages, and Chi-squared and Fisher's exact tests assessed proportional differences. For continuous variables mean \pm SD with two-tailed parametric $(\mathrm{t})$ or nonparametric $(\times 2$, Mann-Whitney) tests were presented. $\mathrm{p}<0.05$ was considered statistically significant.

For repeated sampling, the most recent test result was used, although all data were considered by the multidisciplinary team when deciding final diagnostic outcome (figure 1). Patients with inadequateinconclusive outcomes were excluded for analysis of test accuracy. The sensitivity and specificity of the individual tests were determined firstly based on definite positive or negative diagnostic outcome, and then assuming all valid-inconclusive outcomes to be truly positive or negative (using multidisciplinary diagnosis as a reference standard). Receiver operating characteristic (ROC) curves were constructed for nNO and CBF. Further accuracy analysis was completed using HSVMA and TEM as the reference standards.

Additionally, sensitivity, specificity and predictive values were calculated for those who underwent all diagnostic tests $(n=180)$ and compared with the whole study population (using multidisciplinary diagnosis as a reference standard); the whole population was then further stratified into: 1) the full protocol at UHS or 2) partial protocol when samples were couriered to UHS (nNO measurements not taken). We also allowed for the fact that those aged $<5$ years did not have $\mathrm{nNO}$ readings measured.

For those who underwent all three tests $(n=180)$, theoretical combination testing approaches [22] were used to determine net sensitivity and specificity of simultaneous (two or more tests in parallel; positive result if any test was abnormal) and sequential (second test only performed if first test(s) abnormal) diagnostic protocols. Net sensitivity/specificity used the addition rule of probability for simultaneous tests and the multiplication rule of probability for sequential tests.

\section{Results}

\section{Study population}

We assessed 868 patients between April 2007 and December 2013 (48\% male; median (range) age 7 (0-79) years). 517 (60\%) attended the UHS clinic in person (figure 2a) and 351 samples were delivered by courier (figure $2 \mathrm{~b}) .75$ (9\%) patients had a positive diagnosis, $566(65 \%)$ had a negative diagnosis and 13 (1\%) had inconclusive diagnostic outcome despite adequate samples. 214 (25\%) patients had invalid-inconclusive results due to inadequate data at the time of the study, of whom 113 (13\%) patients had only TEM samples sent from satellite clinics. Invalid-inconclusive outcomes were excluded from the analyses, resulting in a study population of 654, of which 641 had a definitive positive or negative outcome. The characteristics of the positive, negative and inconclusive patients are shown in table 1.

\section{Accuracy of individual diagnostic tests}

Analysis was dependent on the quality of the sample and many patients required repeat biopsies: $17 \%$ (113 out of 654 ) required one repeat; $2 \%$ (11 out of 654 ) required two repeats; and $0.4 \%$ (three out of 654 ) required three repeats. Analysis of diagnostic accuracy was based on the final successful test completed.

\section{Nasal nitric oxide}

nNO was measured in 301 (47\%) patients with a positive or negative diagnosis. nNO was significantly lower in PCD-positive patients $\left(17 \pm 20 \mathrm{~nL} \cdot \mathrm{min}^{-1}, 95 \% \mathrm{CI} 10-23 \mathrm{~nL} \cdot \mathrm{min}^{-1}\right)$ than negative patients $(172 \pm 94$ $\mathrm{nL} \cdot \mathrm{min}^{-1}, 95 \%$ CI $\left.160-183 \mathrm{~nL} \cdot \mathrm{min}^{-1}\right)(\mathrm{p}<0.0001)$ (online supplementary figure $\mathrm{S} 1$ ). ROC curve analysis showed low nNO to be a strong predictor of a multidisciplinary diagnosis of PCD (area under the curve $0.97,95 \%$ CI $0.94-1.00$ ) (figure 3). A cut-off of $30 \mathrm{~nL} \cdot \mathrm{min}^{-1}$ was sensitive (0.91, 95\% CI 0.76-0.98) and specific $(0.96,95 \%$ CI $0.93-0.98)$ (table 2). Inclusion of eight valid-inconclusive results as PCD-positive $\left(109.7 \pm 119 \mathrm{~nL} \cdot \mathrm{min}^{-1}, 95 \% \mathrm{CI} 10-209 \mathrm{~nL} \cdot \mathrm{min}^{-1}\right.$ ) reduced sensitivity to 0.78 (95\% CI 0.62-0.89) (online supplementary table $\mathrm{S} 1$ ). 

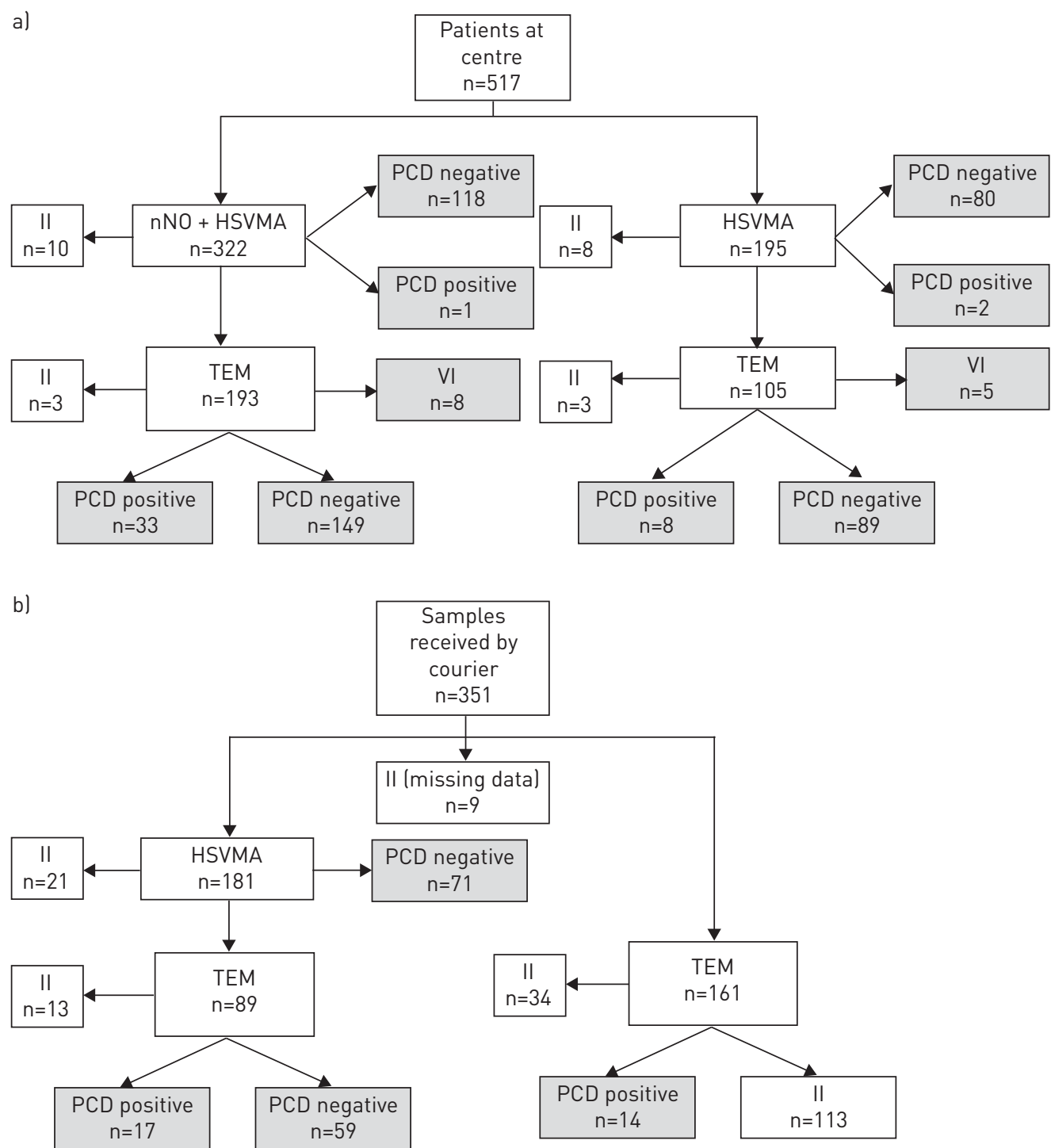

FIGURE 2 The diagnostic investigations and outcomes of patients seen a) at the diagnostic centre at University Hospital Southampton (UHS) or b) having had samples sent by courier to UHS from a satellite respiratory clinic. Patients were diagnosed as primary ciliary dyskinesia (PCD)-positive, PCD-negative or valid-inconclusive (VI). Invalid-inconclusive (II) results due to inadequate samples or data are shown, but were subsequently excluded from accuracy analyses. Diagnostic tests included nasal nitric oxide (nNO), high-speed video microscopy analysis (HSVMA) and transmission electron microscopy (TEM).

High-speed video microscopy analysis

HSVMA was performed in 625 (98\%) patients including 60 PCD-positive and 565 PCD-negative cases. HSVMA was abnormal in all 60 positive patients tested. Of 565 PCD-negative patients, 39 had abnormal or equivocal HSVMA results (17 had abnormal CBF and 22 had abnormalities of CBP). HSVMA had excellent sensitivity (1.00, 95\% CI $0.94-1.00)$ and specificity $(0.93,95 \%$ CI $0.91-0.95)$ (table 2 ). Since our definition of PCD includes abnormal ciliary function (pattern or frequency), sensitivity would be expected to approach 1.00. Inclusion of valid-inconclusive results as PCD-positive kept sensitivity high at 0.97 ( $95 \%$ CI 0.90-1.00) (online supplementary table S1).

Subgroup analysis (e.g. UHS versus courier-delivered or age $<5$ years) made little difference to the sensitivity, specificity or predictive values (tables 2 and 3 ).

The mean CBF for PCD-positive patients $(2.3 \pm 5.2 \mathrm{~Hz}, 95 \%$ CI $0.4-4.3 \mathrm{~Hz})$ was significantly lower than for PCD-negative patients $(15.4 \pm 2.3 \mathrm{~Hz}, 95 \%$ CI $15.2-15.6 \mathrm{~Hz})(\mathrm{p}<0.0001)$ (online supplementary figure S2). ROC curve analysis showed CBF to discriminate well between PCD-positive and -negative patients (AUC 0.92, 95\% CI 0.79-1.00) (figure 3). However, it was not possible to derive a reliable CBF for 31 (41\%) PCD-positive patients with variable CBP. 
TABLE 1 Clinical characteristics of the referral population grouped by positive, negative, valid-inconclusive and invalid-inconclusive diagnostic outcomes

\begin{tabular}{|c|c|c|c|c|c|c|c|c|c|c|c|c|c|c|c|}
\hline & \multicolumn{3}{|c|}{ Total referrals } & \multicolumn{3}{|c|}{ Positive } & \multicolumn{3}{|c|}{ Negative } & \multicolumn{3}{|c|}{ Valid-inconclusive } & \multicolumn{3}{|c|}{ Invalid-inconclusive } \\
\hline & Yes & No & NA $^{\#}$ & Yes & No & NA $^{\#}$ & Yes & No & NA $^{\#}$ & Yes & No & $\mathrm{NA}^{\#}$ & Yes & No & NA $^{\#}$ \\
\hline Subjects & & 868 & & & 75 & & & 566 & & & 13 & & & 214 & \\
\hline Male & $362(42)$ & $393(45)$ & $11(13)$ & $34(45)$ & $36(48)$ & $5(7)$ & 249 (44) & 278 (49) & 39 (7) & $7(54)$ & $6(46)$ & $0(0)$ & 72 (34) & $73(34)$ & 69 (32) \\
\hline Full-term gestation & $346(40)$ & 102 (12) & $420(48)$ & $66(88)$ & $7(9)$ & $2(3)$ & $242(43)$ & $80(14)$ & 244 (43) & $8(61)$ & 2 (15) & $3(23)$ & $30(14)$ & $13(6)$ & $171(80)$ \\
\hline Sibling with PCD & $32(4)$ & 792 (91) & 44 (5) & $18(24)$ & $55(73)$ & $2(3)$ & $9(2)$ & 529 (93) & $28(5)$ & $0(0)$ & 11 (85) & $2(15)$ & $5(2)$ & 197 (92) & $12(6)$ \\
\hline Neonatal unit & $138(16)$ & $694(80)$ & $35(4)$ & $46(61)$ & 25 (33) & $4(5)$ & 77 (14) & $466(82)$ & $23(4)$ & 3 (23) & $9(69)$ & $1(8)$ & $12(6)$ & 194 (91) & $8(4)$ \\
\hline Situs abnormality & $70(8)$ & 788 (91) & $10(1)$ & $33(44)$ & $42(56)$ & $0(0)$ & $22(5)$ & 537 (95) & $7(0)$ & $4(31)$ & 9 (69) & $0(0)$ & $11(5)$ & 200 (93) & $3(1)$ \\
\hline Cardiac abnormality & $20(2)$ & $848(98)$ & $0(0)$ & $6(8)$ & 69 (92) & $0(0)$ & $10(2)$ & 556 (98) & $0(0)$ & $0(0)$ & $13(100)$ & $0(0)$ & $4(2)$ & 210 (98) & $0(0)$ \\
\hline Pulmonary symptoms & $710(82)$ & $158(18)$ & $0(0)$ & $72(96)$ & $3(4)$ & $0(0)$ & $488(86)$ & $78(14)$ & $0(0)$ & 12 (93) & $1(7)$ & $0(0)$ & $138(64)$ & $76(36)$ & $0(0)$ \\
\hline Rhinitis & 477 (55) & $389(45)$ & $2(0)$ & $61(81)$ & 14 (19) & $0(0)$ & 325 (57) & $239(42)$ & $2(1)$ & $9(69)$ & $4(31)$ & $0(0)$ & 82 (38) & $132(62)$ & $0(0)$ \\
\hline Sinusitis & $192(22)$ & $663(76)$ & $13(2)$ & $21(28)$ & $53(71)$ & $1(1)$ & $138(24)$ & $416(74)$ & $12(2)$ & $5(38)$ & $8(62)$ & $0(0)$ & $28(13)$ & 186 (87) & $0(0)$ \\
\hline
\end{tabular}

Data are presented as $n$ or $n(\%)$. Patients with invalid-inconclusive outcomes were excluded from the study population for analyses. NA: not available; PCD: primary ciliary dyskinesia. \#: for example, children did not know their fertility status; older adults did not know neonatal details; and in a minority of cases the data were simply not recorded. 


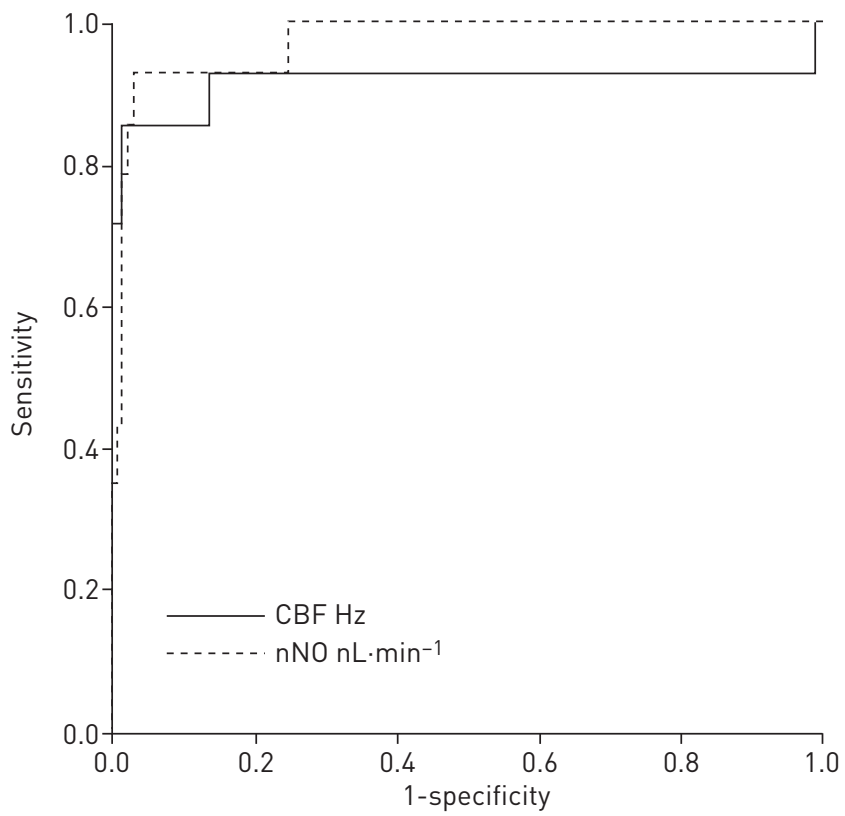

FIGURE 3 Receiver operating characteristic (ROC) curve analysis for ciliary beat frequency (CBF) and nasal nitric oxide (nNO) for predicting a diagnosis of primary ciliary dyskinesia (PCD) lusing multidisciplinary diagnosis as the reference standard). ROC curve analysis showed that $\mathrm{nNO} \leqslant 30 \mathrm{~nL} \cdot \mathrm{min}^{-1}$ larea under the curve (AUC) $0.97,95 \% \mathrm{Cl} 0.94-1.00$ ) was superior to CBF (AUC $0.92,95 \% \mathrm{Cl} 0.79-1.00$ ) as predictors of a PCD-positive diagnosis.

\section{Transmission electron microscopy}

TEM was performed on samples from 368 (57\%) patients including 72 PCD-positive and 297 PCD-negative cases. 57 (79\%) out of 72 PCD-positive patients had hallmark ultrastructural defects of PCD: $31 \%$ with outer dynein arm (ODA) and inner dynein arm (IDA) defects; $26 \%$ with an ODA defect; $10 \%$ with an ODA defect and suspected IDA defect; $7 \%$ with microtubule disarrangement and IDA defect; $4 \%$ with an intermittent central pair microtubule defect; and $1 \%$ with a microtubule transposition defect. $21 \%$ had "normal" ciliary ultrastructure. None of the 297 PCD-negative patients had ultrastructural changes suggestive of PCD, but secondary changes (e.g. swollen membranes or compound cilia) were fairly frequent.

TEM sensitivity was 0.79 (95\% CI 0.68-0.88) and specificity was 1.0 (95\% CI 0.99-1.00) (table 2). Again, subgroup analysis made little difference to the sensitivity, specificity or predictive values (tables 2 and 3 ).

TABLE 2 The diagnostic accuracy of nasal nitric oxide (nNO), high-speed video microscopy analysis (HSVMA) and transmission electron microscopy (TEM) analysis to diagnose primary ciliary dyskinesia

\begin{tabular}{lccc} 
& nNO $\leqslant \mathbf{3 0 ~} \mathbf{~ n L} \cdot \mathbf{m i n}^{-1}$ & HSVMA & TEM \\
\hline Subjects $^{\#}$ & $301(47)$ & $625(98)$ & $368(57)$ \\
Positive patients $^{\text {I }}$ & $34(45)$ & $60(80)$ & $71(95)$ \\
Negative patients $^{+}$ & $267(47)$ & $565(100)$ & $297(52)$ \\
True positive & 31 & 60 & 56 \\
True negative & 257 & 526 & 297 \\
False positive & 10 & 39 & 0 \\
False negative & 3 & 0 & 15 \\
Sensitivity (95\% CI) & $0.91(0.76-0.98)$ & $1.00(0.94-1.00)$ & $0.79(0.68-0.88)$ \\
Specificity (95\% CI) & $0.96(0.93-0.98)$ & $0.93(0.91-0.95)$ & $1.00(0.99-1.00)$ \\
PPV (95\% CI) & $0.76(0.60-0.88)$ & $0.61(0.50-0.70)$ & $1.00(0.94-1.00)$ \\
NPV (95\% CI) & $0.99(0.97-1.00)$ & $1.00(0.99-1.00)$ & $0.95(0.92-0.97)$
\end{tabular}

Data are presented as $\mathrm{n}(\%)$ or $\mathrm{n}$, unless otherwise stated. Data were analysed for patients with conclusive positive or negative results who underwent the individual tests. PPV: positive predictive value; NPV: negative predictive value. ${ }^{\#}: \mathrm{n}=641 ;{ }^{\text {ๆ}}: \mathrm{n}=75 ;{ }^{+}: \mathrm{n}=566$. 
Air-liquid interface culture

ALI culture was performed on 808 samples and 241 (30\%) ciliated. Ciliary function was reanalysed following ALI culture in 152 (24\%) patients. ALI samples confirmed a persistent abnormality of CBP in 21 out of 21 PCD-positive patients. Out of 124 PCD-negative patients, 123 had a normal CBP following ALI culture, and one patient had uncoordinated cilia, perhaps due to variable cell health.

\section{Accuracy of combinations of tests}

Various combinations of diagnostic tests are undertaken at our centre (figure 2) while alternative combinations are used in other centres [23]. We calculated accuracy for the combinations of tests in 180 patients who had undergone all diagnostic tests, allowing us to consider theoretical scenarios (table 4). If $\mathrm{nNO}$ had been used as a screening test followed sequentially by TEM, 36 patients would have proceeded to TEM, but three out of 31 PCD patients would have been "missed" by not proceeding to further testing due to false negative nNO results; TEM would have subsequently failed to identify nine PCD patients. The net specificity for this combination of tests was excellent (100\%), but net sensitivity was poor, failing to identify PCD in 12 (39\%) out of 31 patients. Alternatively, if $\mathrm{nNO}$ had been used as a screening test followed sequentially by HSVMA, three out of 31 PCD patients would not have proceeded to HSVMA; however, HSVMA would have subsequently identified all 28 positive patients. Therefore, the net sensitivity and specificity were $90 \%$ and $100 \%$, respectively.

Excellent net sensitivity and specificity were achieved upon simultaneous testing of HSVMA with nNO ( $100 \%$ and $87 \%$, respectively) or HSVMA with TEM (100\% and $92 \%$, respectively) or all three tests $(100 \%$ and $87 \%$, respectively).

\section{Accuracy of individual tests using HSVMA or TEM as the reference standard}

We calculated the accuracies of individual tests assuming HSVMA and TEM to be the reference standard for diagnosing PCD. When TEM analysis was considered as the reference standard, HSVMA sensitivity and specificity were 1.00 and 0.86 , respectively, and $\mathrm{nNO}\left(\leqslant 30 \mathrm{~nL} \cdot \mathrm{min}^{-1}\right)$ sensitivity and specificity were 0.95 and 0.89 , respectively. When HSVMA was considered as the reference standard, TEM sensitivity was 0.48 (95\% CI $0.38-0.59)$ and specificity was 1.00 (95\% CI $0.98-1.00)$; $\mathrm{nNO}\left(\leqslant 30 \mathrm{~nL} \cdot \mathrm{min}^{-1}\right)$ sensitivity was 0.50 and specificity was 0.96 (online supplementary table S2).

\section{Discussion}

Our large cross-sectional study provides prospectively collected outcome data following a comprehensive range of PCD diagnostic tests. Our diagnostic algorithm varies from some centres [23], but the findings may contribute to the development of international consensus.

A strength of this study was analyses of consecutive referrals within a national diagnostic programme, as this is likely to yield the most valid estimates of diagnostic accuracy. Although not all patients underwent all tests, this pragmatic study reflects the real patient journey. The major limitation is the lack of a "gold reference standard"; we therefore used a surrogate standard of expert multidisciplinary consensus. Since each test contributes to the final decision, sensitivity and specificity might be overestimated. Additionally, genetic testing does not currently form part of our diagnostic pathway and this is a rapidly expanding area that is used for diagnosis in many countries.

$11.5 \%$ of patients with adequate samples were diagnosed as PCD-positive, which is slightly lower than some centres $[6,8,24]$, but is similar to or higher than others (Switzerland, Amsterdam and London (Claudia Kuehni, Institute of Social and Preventive Medicine, University of Bern, Bern, Switzerland; Eric Haarman, VU University Medical Center, Amsterdam, the Netherlands; and Claire Hogg, Royal Brompton \& Harefield NHS Foundation Trust, London UK; personal communications). Higher positive rates may be seen at centres with $\mathrm{nNO}$ prescreening, in consanguineous populations [25] or if access to testing is restricted to those who are extremely likely to have PCD. The prevalence in referral populations will not affect the sensitivity or specificity but will alter the positive and negative predictive values of the tests.

\section{High-speed video microscopy analysis}

HSVMA was sensitive and specific for diagnosing PCD; however, if used as a reference standard, this would lead to a high number of false positive results. In line with many European centres we consider HSVMA a first-line test, which might inflate the sensitivity; this needs further investigation in blinded studies. Since HSVMA is a qualitative test with potential subjectivity, results are regularly validated by external experts, but this does not exclude the possibility of some false negative findings. PCD-negative patients all had predominantly normal HSVMA, but often included a proportion of dyskinetic cilia probably due to recent infection or damage during sampling [26]. Some PCD patients had areas of 
TABLE 3 The diagnostic accuracy of nasal nitric oxide (nNO), high-speed video microscopy analysis (HSVMA) and transmission electron microscopy (TEM) analysis to diagnose primary ciliary dyskinesia

UHS

Courier

\begin{tabular}{|c|c|c|c|c|c|c|c|c|c|}
\hline & \multirow{2}{*}{\multicolumn{2}{|c|}{$<5$ years }} & \multirow{2}{*}{\multicolumn{3}{|c|}{$>5$ years }} & \multirow{2}{*}{\multicolumn{2}{|c|}{$<5$ years }} & \multirow{2}{*}{\multicolumn{2}{|c|}{$>5$ years }} \\
\hline & & & & & & & & & \\
\hline & HSVMA & TEM & $\mathrm{nNO} \leqslant 30 \mathrm{~nL} \cdot \mathrm{min}^{-1}$ & HSVMA & TEM & HSVMA & TEM & HSVMA & TEM \\
\hline Subjects & \multicolumn{2}{|c|}{126} & \multicolumn{3}{|c|}{355} & \multicolumn{2}{|c|}{80} & \multicolumn{2}{|c|}{80} \\
\hline Total & 125 (99) & $67(53)$ & $301(85)$ & 353 (99) & $212(60)$ & 71 (89) & $48(60)$ & 76 (95) & $41(51)$ \\
\hline Positive & $8(6)$ & $7(10)$ & $34(11)$ & $34(10)$ & $33(16)$ & $8(11)$ & 17 (35) & $10(13)$ & $14(34)$ \\
\hline Negative & $117(94)$ & $60(90)$ & $267(89)$ & $319(90)$ & $179(84)$ & $63(89)$ & $31(65)$ & $66(87)$ & $27(66)$ \\
\hline True positive & 8 & 6 & 31 & 34 & 22 & 8 & 15 & 10 & 13 \\
\hline True negative & 111 & 60 & 257 & 297 & 179 & 59 & 31 & 59 & 27 \\
\hline False positive & 6 & 0 & 10 & 22 & 0 & 4 & 0 & 7 & 0 \\
\hline False negative & 0 & 1 & 3 & 0 & 11 & 0 & 2 & 0 & 1 \\
\hline Sensitivity (95\% CI) & $1.00(0.63-1.00)$ & $0.86(0.42-0.98)$ & $0.91(0.76-0.98)$ & $1.00(0.90-1.00)$ & $0.67(0.48-0.82)$ & $1.00(0.63-1.00)$ & $0.88(0.64-0.98)$ & $1.00(0.69-1.00)$ & $0.93(0.66-0.99)$ \\
\hline Specificity $(95 \%$ CI) & $0.95(0.89-0.98)$ & $1.00(0.94-1.00)$ & $0.96(0.93-0.98)$ & $0.93(0.90-0.96)$ & $1.00(0.98-1.00)$ & $0.94(0.85-0.98)$ & $1.00(0.89-1.00)$ & $0.89(0.79-0.96)$ & $1.00(0.87-1.00)$ \\
\hline PPV $(95 \%$ CI) & $0.57(0.29-0.82)$ & $1.00(0.54-1.00)$ & $0.76(0.60-0.88)$ & $0.61(0.47-0.74)$ & $1.00(0.84-1.00)$ & $0.67(0.35-0.90)$ & $1.00(0.78-1.00)$ & $0.59(0.33-0.81)$ & $1.00(0.75-1.00)$ \\
\hline NPV $(95 \% \mathrm{CI})$ & $1.00(0.97-1.00)$ & $0.98(0.91-1.00)$ & $0.99(0.97-1.00)$ & $1.00(0.99-1.00)$ & $0.94(0.90-0.97)$ & $1.00(0.94-1.00)$ & $0.94(0.80-0.99)$ & $1.00(0.94-1.00)$ & $0.96(0.82-0.99)$ \\
\hline
\end{tabular}

Data are presented as $\mathrm{n}$ or $\mathrm{n}(\%)$, unless otherwise stated. Analyses were stratified by patients seen at University Hospital Southampton (UHS) and samples sent by courier to UHS; then stratified further by age $<5$ years and $\geqslant 5$ years at time of assessment. PPV: positive predictive value; NPV: negative predictive value.

TABLE 4 Sensitivity and specificity of high-speed video microscopy analysis (HSVMA), ciliary beat pattern, nasal nitric oxide (nNO) and transmission electron microscopy (TEM) applied as single or combined tests, using simultaneous or sequential testing

\begin{tabular}{|c|c|c|c|c|c|c|c|c|c|c|}
\hline & \multicolumn{3}{|c|}{ Single testing } & \multicolumn{3}{|c|}{ Simultaneous testing } & \multicolumn{4}{|c|}{ Sequential two-stage testing } \\
\hline & nNO & HSVMA & TEM & nNO + HSVMA & HSVMA + TEM & nNO + HSVMA + TEM & $\begin{array}{l}\text { 1. } \mathrm{nNO}^{\#} \\
\text { 2. HSVMA" }\end{array}$ & $\begin{array}{l}\text { 1. } \mathrm{nNO}^{\#} \\
\text { 2. } \mathrm{TEM}^{\Uparrow}\end{array}$ & $\begin{array}{c}\text { 1. } \mathrm{nNO}+\mathrm{HSVMA}^{\#} \\
\text { 2. } \mathrm{TEM}^{+}\end{array}$ & $\begin{array}{l}\text { 1. } \text { HSVMA }^{\#} \\
\text { 2. } \text { TEM }^{\S}\end{array}$ \\
\hline Subjects & 180 & 180 & 180 & 180 & 180 & 180 & 36 & 36 & 51 & 43 \\
\hline Positive & 31 & 31 & 31 & 31 & 31 & 31 & 28 & 28 & 31 & 31 \\
\hline Negative & 149 & 149 & 149 & 149 & 149 & 149 & 8 & 8 & 20 & 12 \\
\hline True positive & 28 & 31 & 20 & 31 & 31 & 31 & 28 & 19 & 20 & 20 \\
\hline True negative & 141 & 137 & 149 & 129 & 137 & 129 & 8 & 8 & 20 & 12 \\
\hline False positive & 8 & 12 & 0 & 20 & 12 & 20 & 0 & 0 & 0 & 0 \\
\hline False negative & 3 & 0 & 11 & 0 & 0 & 0 & 0 & 9 & 11 & 11 \\
\hline Sensitivity $(95 \% \mathrm{CI})$ & $0.90(0.74-0.98)$ & $1.00(0.89-1.00)$ & $0.65(0.45-0.81)$ & & & & & & & \\
\hline Specificity $(95 \% \mathrm{CI})$ & $0.95(0.90-0.98)$ & $0.92(0.86-0.96)$ & $1.00(0.98-1.00)$ & & & & & & & \\
\hline PPV $(95 \% \mathrm{CI})$ & $0.78(0.61-0.90)$ & $0.72(0.56-0.85)$ & $1.00(0.83-1.00)$ & & & & & & & \\
\hline NPV $(95 \% \mathrm{CI})$ & $0.98(0.94-1.00)$ & $1.00(0.97-1.00)$ & $0.93(0.88-0.97)$ & & & & & & & \\
\hline Net sensitivity $f$ & & & & 1.00 & 1.00 & 1.00 & 0.90 & 0.61 & 0.65 & 0.65 \\
\hline Net specificity ${ }^{f}$ & & & & 0.87 & 0.92 & 0.87 & 1.00 & 1.00 & 1.00 & 1.00 \\
\hline
\end{tabular}

Data are presented as $n$, unless otherwise stated. PPV: positive predictive value; NPV: negative predictive value. ${ }^{\#}: n=180 ;{ }^{\uparrow}: n=36 ;{ }^{+}: n=51 ;{ }^{\S}: n=43 ;{ }^{f}:$ the net sensitivity and specificity were calculated for combined tests. 
apparently normal ciliary function, highlighting that PCD scientists require significant experience of the qualitative and quantitative range of PCD and non-PCD samples.

HSVMA standardisation is challenging, and robust training, data validation, external audit and continued learning is in place. Our data cannot be generalised to centres where different standards apply. HSVMA requires expensive high-speed video equipment, high optical magnification and digital resolution for accurate $\mathrm{CBP}$ analysis, without which errors are likely. $\mathrm{CBF}$ is $\mathrm{pH}$ and temperature dependent, and we conduct analyses using $\mathrm{pH}$-stable medium equilibrated to $37^{\circ} \mathrm{C}[27,28]$.

Inadequate samples and inconclusive results were a common issue for HSVMA. Recent reports of PCD-causing genes associated with subtle changes at HSVMA and TEM [29, 30] have confirmed our suspicion that some inconclusive results might represent disease. Moreover, samples providing inadequate cilia may be caused by mutations causing a syndrome similar to PCD associated with sparse but normal cilia $[31,32]$. It is possible that some patients excluded from analyses due to inadequate samples will fall into this category. However, in our experience, inadequate samples are commonly adequate upon repeat brushing following antibiotics and when patients are well. We always recommend repeat testing for inadequate samples; some invalid-inconclusive cases did not return for testing because symptoms had resolved or an alternative diagnosis was identified.

\section{Transmission electron microscopy}

Approximately a fifth of PCD patients had apparently normal ciliary ultrastructure, confirming that TEM is unreliable in isolation $[21,33$. However, it is a vital part of the diagnostic portfolio, supporting HSVMA findings and providing a diagnosis when HSVMA is not available or inconclusive. Analysis of ciliary ultrastructure requires expensive equipment and electron microscopists experienced in the range of normality and abnormality. Some abnormalities are straightforward (e.g. ODA defect), but we have diagnosed several patients with subtle abnormalities of microtubules, supported by nNO and HSVMA, that would only be detected by an experienced microscopist analysing sufficient numbers of cilia in transverse and longitudinal section.

\section{Nasal nitric oxide}

$\mathrm{nNO}$ is a recommended screening test for symptomatic patients $[5,6,34]$. At the outset of the prospective data collection in 2007, a cut-off of $30 \mathrm{~nL} \cdot \mathrm{min}^{-1}$ was arbitrarily set based on prior experience. Recent evidence suggests that higher cut-offs may be more useful $[4,34]$, and the accuracy of nNO cut-offs for screening/diagnostics needs to be standardised based on emerging evidence. $30 \mathrm{~nL} \cdot \mathrm{min}^{-1}$ was used clinically throughout data collection and so it is on this basis that we have analysed the data. The sensitivity and specificity of this cut-off were 0.91 and 0.96 , respectively. Therefore, $9 \%$ of cases might be missed if further testing was excluded on the basis of this test in isolation. $77 \mathrm{~nL} \cdot \mathrm{min}^{-1}$ has recently been recommended as a cut-off [4]; this cut-off improved sensitivity in our population to $96 \%$, but reduced specificity to $83 \%$. In LEIGH et al.'s [4] study, sensitivity to detect patients with PCD diagnosed by TEM or genetics was 0.98 while specificity was $>0.75$, similar to our findings. In our centre we are confident to use a cut-off of $30 \mathrm{~nL} \cdot \mathrm{mL}^{-1}$ because it is always alongside a HSVMA result. In our opinion, if nNO is used by referral centres to decide who to refer for testing, the higher cut-off with greater sensitivity should be used, but it is notable that $4 \%$ of cases might still be missed. We use nNO to support a positive diagnosis in patients with consistent subtle abnormalities of CBP who might otherwise be labelled inconclusive. We would be cautious to exclude a diagnosis of PCD in patients with $\mathrm{nNO} \leqslant 30 \mathrm{~nL} \cdot \mathrm{min}^{-1}$, and these patients are more likely to be considered inconclusive and therefore undergo repeated testing. Only $47 \%$ of the study population underwent nNO testing, because it is not available at satellite centres and the breath-hold manoeuvre is usually technically acceptable only in those aged $>5$ years. However, the present article reports nNO data from 301 participants, which constitutes the largest study to date in a PCD diagnostic clinic population.

\section{Accuracy of combinations of tests}

Data from patients who had undergone all tests $(n=180)$ were used to calculate the accuracy for possible combinations of tests. Two-stage testing based on $\mathrm{nNO}$ prescreening followed by TEM potentially missed $\sim 40 \%$ of PCD cases, because both tests were required to be positive for a positive diagnosis [22]. However, our $30 \mathrm{~nL} \cdot \mathrm{min}^{-1}$ cut-off is probably too low for use as a screening threshold [34] and the previously discussed subjectivity of HSVMA needs to be taken into account. Simultaneous testing requires one positive test result for a positive diagnosis and, conversely, all tests to be negative for a negative outcome [22]. Using all three tests simultaneously (where any abnormal test leads to a positive result) sensitivity was $100 \%$, but specificity reduced to $87 \%$, compared to our multidisciplinary approach where no test was considered in isolation ( $100 \%$ sensitivity and $92 \%$ specificity). 


\section{Concluding comments}

Advances in understanding the molecular genetic basis of PCD have been made in recent years, to the extent that genetic testing is now able to detect $\sim 65 \%$ of PCD cases. However, genetic testing for PCD is not yet available in the UK except as a research tool [1]. As more genes are identified, genetic testing by multigene panel [1] will make genotyping a cost-effective approach. Characterisation of ciliary structure and function will continue to have a place within diagnostic processes, similar to the need for functional tests to confirm the diagnosis of cystic fibrosis [35]. Moreover, a thorough definition of disease phenotype by cilia ultrastructure, cilia beat pattern and $\mathrm{nNO}$ production rate will be extremely helpful in guiding genetic analyses in this genetically heterogeneous disease. The English public healthcare system does not fund immunofluorescence staining of ciliary proteins as a diagnostic test. This method is currently only able to detect abnormalities that are evident by TEM, and would therefore not improve our diagnostic accuracy. However, we anticipate that as more antibodies become available, immunofluorescence staining will prove a time- and cost-efficient diagnostic test.

There is no single diagnostic test that can be used universally to diagnose PCD. Recent reports of PCD-causing genes (RSPH1) associated with subtle HSVMA and TEM abnormalities with normal nNO demonstrate the skill and expert microscopists needed for accurate diagnoses $[29,30,36]$.

Importantly, the conduct and reporting of tests used to diagnose PCD are not standardised. We believe that the time is right to develop consensus standards for equipment, staff experience and protocols.

\section{Acknowledgements}

Thanks to Gary Connett, Julian Legg, Kerry Gove and Karen Lock (University Hospital Southampton NHS Foundation Trust, Southampton, UK) for their contribution to clinical assessments.

\section{References}

1 Lucas JS, Burgess A, Mitchison HM, et al. Diagnosis and management of primary ciliary dyskinesia. Arch Dis Child 2014; 99: 850-856.

2 O'Callaghan C, Chilvers M, Hogg C, et al. Diagnosing primary ciliary dyskinesia. Thorax 2007; 62: 656-657.

3 Knowles MR, Daniels LA, Davis SD, et al. Primary ciliary dyskinesia. Recent advances in diagnostics, genetics, and characterization of clinical disease. Am J Respir Crit Care Med 2013; 188: 913-922.

4 Leigh MW, Hazucha MJ, Chawla KK, et al. Standardizing nasal nitric oxide measurement as a test for primary ciliary dyskinesia. Ann Am Thorac Soc 2013; 10: 574-581.

5 Barbato A, Frischer T, Kuehni CE, et al. Primary ciliary dyskinesia: a consensus statement on diagnostic and treatment approaches in children. Eur Respir J 2009; 34: 1264-1276.

6 Marthin JK, Nielsen KG. Choice of nasal nitric oxide technique as first-line test for primary ciliary dyskinesia. Eur Respir J 2011; 37: 559-565.

7 Papon JF, Bassinet L, Cariou-Patron G, et al. Quantitative analysis of ciliary beating in primary ciliary dyskinesia: a pilot study. Orphanet J Rare Dis 2012; 7: 78.

8 Stannard WA, Chilvers MA, Rutman AR, et al. Diagnostic testing of patients suspected of primary ciliary dyskinesia. Am J Respir Crit Care Med 2010; 181: 307-314.

9 Chilvers MA, O'Callaghan C. Analysis of ciliary beat pattern and beat frequency using digital high speed imaging: comparison with the photomultiplier and photodiode methods. Thorax 2000; 55: 314-317.

10 Chilvers MA, Rutman A, O'Callaghan C. Ciliary beat pattern is associated with specific ultrastructural defects in primary ciliary dyskinesia. J Allergy Clin Immunol 2003; 112: 518-524.

11 Papon JF, Coste A, Roudot-Thoraval F, et al. A 20-year experience of electron microscopy in the diagnosis of primary ciliary dyskinesia. Eur Respir J 2010; 35: 1057-1063.

12 Shoemark A, Dixon M, Corrin B, et al. Twenty-year review of quantitative transmission electron microscopy for the diagnosis of primary ciliary dyskinesia. J Clin Pathol 2012; 65: 267-271.

13 Jorissen M, Willems T, Van der Schueren B. Ciliary function analysis for the diagnosis of primary ciliary dyskinesia: advantages of ciliogenesis in culture. Acta Otolaryngol 2000; 120: 291-295.

14 Hirst RA, Jackson CL, Coles JL, et al. Culture of primary ciliary dyskinesia epithelial cells at air-liquid interface can alter ciliary phenotype but remains a robust and informative diagnostic aid. PloS One 2014; 9: e89675.

15 Omran H, Loges NT. Immunofluorescence staining of ciliated respiratory epithelial cells. Methods Cell Biol 2009; 91: $123-133$.

16 Walker WT, Jackson CL, Lackie PM, et al. Nitric oxide in primary ciliary dyskinesia. Eur Respir J 2012; 40: 1024-1032.

17 Marthin JK, Mortensen J, Pressler T, et al. Pulmonary radioaerosol mucociliary clearance in diagnosis of primary ciliary dyskinesia. Chest 2007; 132: 966-976.

18 Lucas JS, Chetcuti P, Copeland F, et al. Overcoming challenges in the management of primary ciliary dyskinesia: the UK model. Paediatr Respir Rev 2014; 15: 142-145.

19 Shinkins B, Thompson M, Mallett S, et al. Diagnostic accuracy studies: how to report and analyse inconclusive test results. BMJ 2013; 346: f2778.

20 Harris A, Bhullar E, Gove K, et al. Validation of a portable nitric oxide analyzer for screening in primary ciliary dyskinesias. BMC Pulm Med 2014; 14: 18

21 Boon M, Smits A, Cuppens H, et al. Primary ciliary dyskinesia: critical evaluation of clinical symptoms and diagnosis in patients with normal and abnormal ultrastructure. Orphanet J Rare Dis 2014; 9: 11.

22 Gordis L. Assessing the validity and reliability of diagnostic and screening tests. In: Gordis L, ed. Epidemiology. 4th Edn. Philadelphia, Elsevier/Saunders, 2009; pp. 85-108. 
23 Lucas JS, Leigh MW. Diagnosis of primary ciliary dyskinesia: searching for a gold standard. Eur Respir J 2014; 44: 1418-1422.

24 Boon M, Meyts I, Proesmans M, et al. Diagnostic accuracy of nitric oxide measurements to detect primary ciliary dyskinesia. Eur J Clin Invest 2014; 44: 477-485.

25 O'Callaghan C, Chetcuti P, Moya E. High prevalence of primary ciliary dyskinesia in a British Asian population. Arch Dis Child 2010; 95: 51-52.

26 Thomas B, Rutman A, O'Callaghan C. Disrupted ciliated epithelium shows slower ciliary beat frequency and increased dyskinesia. Eur Respir J 2009; 34: 401-404.

27 Smith CM, Hirst RA, Bankart MJ, et al. Cooling of cilia allows functional analysis of the beat pattern for diagnostic testing. Chest 2011; 140: 186-190.

28 Jackson CL, Goggin PM, Lucas JS. Ciliary beat pattern analysis below $37^{\circ} \mathrm{C}$ may increase risk of primary ciliary dyskinesia misdiagnosis. Chest 2012; 142: 543-544.

29 Kott E, Legendre M, Copin B, et al. Loss-of-function mutations in RSPH1 cause primary ciliary dyskinesia with central-complex and radial-spoke defects. Am J Hum Genet 2013; 93: 561-570.

30 Castleman VH, Romio L, Chodhari R, et al. Mutations in radial spoke head protein genes RSPH9 and RSPH4A cause primary ciliary dyskinesia with central-microtubular-pair abnormalities. Am J Hum Genet 2009; 84: 197-209.

31 Wallmeier J, Al-Mutairi DA, Chen CT, et al. Mutations in CCNO result in congenital mucociliary clearance disorder with reduced generation of multiple motile cilia. Nat Genet 2014; 46: 646-651.

32 Boon $\mathrm{M}$, Wallmeier J, Ma L, et al. MCIDAS mutations result in a mucociliary clearance disorder with reduced generation of multiple motile cilia. Nat Commun 2014; 5: 4418.

33 Knowles MR, Leigh MW, Carson JL, et al. Mutations of DNAH11 in patients with primary ciliary dyskinesia with normal ciliary ultrastructure. Thorax 2012; 67: 433-441.

34 Collins SA, Gove K, Walker W, et al. Nasal nitric oxide screening for primary ciliary dyskinesia: systematic review and meta-analysis. Eur Respir J 2014; 44: 1589-1599.

35 Hogg C, Bush A. Genotyping in primary ciliary dyskinesia: ready for prime time, or a fringe benefit? Thorax 2012; 67: 377-378.

36 Knowles MR, Ostrowski LE, Leigh MW, et al. Mutations in RSPH1 cause primary ciliary dyskinesia with a unique clinical and ciliary phenotype. Am J Respir Crit Care Med 2014; 189: 707-717. 